\title{
An automatic procedure for induction motor parameter estimation at standstill
}

\author{
Luca Peretti*, Associate Member, IET, and Mauro Zigliotto**, Member, IET \\ * ABB Corporate Research Center, Västerås, Sweden (e-mail: luca.peretti@se.abb.com). \\ ** University of Padova, Department of Technique and Management of Industrial Systems, Vicenza, Italy (email: \\ mauro.zigliotto@unipd.it)
}

\begin{abstract}
The paper presents a self-commissioning procedure for the automatic parameter estimation of three-phase induction motor drives. The procedure consists of a step-by-step approach with different test signals to obtain the parameter values, while maintaining the motor at standstill. The actual implementation is capable of mapping both inverter and motor parameters nonlinearities, providing accurate data for the tuning of common current regulators and for advanced sensorless drives as well. Theoretical and experimental results are provided, proving the effectiveness of the procedure.
\end{abstract}

Index Terms-AC converter machines, Induction motors, Parameter estimation, Non-linear models, Digital control.

\section{INTRODUCTION}

Parameter estimation of $\mathrm{AC}$ machines is a research topic in the electric drive area for almost three decades. From the early scalar controllers to the more recent vector field-oriented control (FOC), a precise knowledge of motor parameters has always been a key issue.

Induction motor (IM) parameter estimation represents one of the most widely studied topics in the electric drive literature, mainly due to the widespread use of IMs in industry. Early papers, as for example [1], were devoted to parameter sensitivity analysis. Then, many papers discussed how to provide constant motor parameters to FOC algorithms for IMs. Among the presented solutions, [2] and [3] are quite representative. In [2], one of the first standstill procedures for IM parameter estimation was presented. The procedure exploits DC and transient measurements on currents and references voltages from the controllers to estimate the stator resistance, the leakage inductance and the rotor time constant of the machine under test. As mentioned by the Author, the procedure is still limited to linear models. Magnetic saturation is not considered, even for the calculation of the rotor time constant, which is obtained through specific transient tests that do not saturate the machine. A more comprehensive solution for parameter estimation of FOC drives is presented in [3], which claims a complete drive tuning within $60 \mathrm{~s}$. However, the motor rotates during the tuning process. Moreover, it requires a speed sensor and a phase voltage measurement, which is not common in industrial drives. The same solution is recalled in [4], where the parameter estimation is performed at standstill using a PWM inverter and injecting DC and sinusoidal currents. Major

L. Peretti was formerly with the University of Padova, Department of Technique and Management of Industrial Systems, Vicenza, Italy. drawbacks are related to the simplifications introduced in the estimation equations and, again, the supposed linearity of the system.

Recently, and using a similar approach, [5] presented a very simple and handy standstill test. The paper claims that two standstill impedance measurements, using two sinusoidal voltage signal injections, are mathematically sufficient to determine the IM parameters of the well-known inverse- $\Gamma$ fourelements equivalent circuit. The solution is to be considered as the simplest one in the scenario, although the least accurate. A slightly more precise procedure, based on two sinusoidal signal injection, is reported in [6]. The paper includes a detailed discussion about the frequency dependence of some of the motor parameters, but the problem of inductance saturation is not solved yet.

More recently, a research branch focused on the realization of reliable sensorless drives, in which AC motors are electronically speed-driven without the need of a position/speed sensor, which is substituted by a mathematical speed observer that heavily relies on the motor model. An accurate knowledge of model parameters is mandatory for a stable sensorless drive with acceptable performances. All in all, this implies that parameter non-linearities, like magnetic saturation, should be included.

Two different approaches exist to track parameter nonlinearities. The first approach is the on-line tracking of the most critical parameters of the IM like stator and rotor resistances, in order to update on-the-fly the tuning of sensorless algorithms. Interesting solutions have been presented in [7], [8] and [9].

The second approach makes use of off-line procedures, which use test signals to compute a detailed map of IM parameters non-linearities, prior or during the final commissioning phase. [10] proposed a standstill solution which takes into account the magnetising inductance variation. The flux linkage-current relationship is estimated with a third-order polynomial by means of a sinusoidal current injection and a recursive least square algorithm. However, the procedure needs a prior knowledge of other IM parameters. In [11], a fullycomprehensive series of laboratory tests has been performed on an IM, with the aim of obtaining a complete description of the motor parameters. As a matter of fact, an excellent self-commissioning scheme should obtain results as close as possible as the ones shown in that work. Procedures like that are not suitable for self-commissioning, since they are not 
executed at standstill and they need a varying mechanical load. They also require a quite high computational effort. However, results clearly show the dependence and the non-linearities of every IM parameter with respect to IM state variables (for example, the magnetising inductance as function of the magnetising current).

This work presents a complete off-line self-commissioning procedure for IM parameter estimation at standstill. The algorithm is not limited to linear models and it improves the results obtainable with the procedures of [12], [13] and [14], by innovating and merging them all with a step-bystep approach. The setup is composed by a conventional induction motor fed by a three-phase PWM inverter. Several different test signals are used in sequence, with the aim of extracting and mapping parameter non-linearities, as proven in the experimental section. The procedure is performed at standstill, and without any change in motor connections, so that it fits for motors already placed on site, too. When the machine is supplied from an inverter, there will appear high frequency components of the current, superimposed to the sinusoidal signals. These higher harmonics are of few interest here, since the goal is to get a model valid for the first harmonic of the supply. Actually, this makes sense since any vector controller (and sensorless scheme as well) operates to control fundamental quantities only [15]. Of course, a model valid also at high frequency could improve the accuracy in fast dynamic responses (e.g. step response) but the trade-off is with a unitary, simple and easy to handle lumped-parameter model. The limited improvement in the accuracy that derives from the modelling of high-frequency effects on parameters would hardly justify the rise of complexity of the model topology.

The paper is organised as follows: Sect. II recalls the basics of the induction motor, with particular focus on the magnetically saturated machines and their equivalent circuit representation at standstill. Sect. III details the theory and the sequence of the step-by-step procedure for each parameter of the machine. Sect. IV shows some experimental results obtained from the implementation of the procedure in the laboratory, by using a fast-control prototyping test bench. Considerations and comments on the obtained results are given as well, before the conclusive remarks of Sect. V.

\section{INDUCTION MOTOR BASICS}

The general IM space vector voltage balance equations for a symmetric machine can be expressed in the stationary $\alpha \beta$ reference as follows [16]:

$$
\left\{\begin{aligned}
\boldsymbol{u}_{s} & =R_{s} \boldsymbol{i}_{s}+\frac{d \boldsymbol{\lambda}_{s}}{d t} \\
\mathbf{0} & =R_{s r} \boldsymbol{i}_{s r}+\frac{d \boldsymbol{\lambda}_{s r}}{d t}-j \omega_{m e} \boldsymbol{\lambda}_{s r}
\end{aligned}\right.
$$

In (1), the space vectors are relative to the stator phase voltages $\left(u_{s a}, u_{s b}, u_{s c}\right)$ and currents $\left(i_{s a}, i_{s b}, i_{s c}\right)$, the rotor currents referred to the stator $\left(i_{s r a}, i_{s r b}, i_{s r c}\right)$, the stator flux linkages $\left(\lambda_{s a}, \lambda_{s b}, \lambda_{s c}\right)$ and the rotor flux linkages referred to the stator, $\left(\lambda_{s r a}, \lambda_{s r b}, \lambda_{s r c}\right) . R_{s}$ is the stator resistance, $R_{s r}$ is the rotor resistance referred to the stator, and $\omega_{m e}$ is the electromechanical speed, which is equal to $p \omega_{m}$ where $p$ is the pole pair and $\omega_{m}$ is the mechanical speed. Further expansions of (1) require some additional considerations, reported below.

\section{A. Magnetically saturated machines}

The constitutive equation of a generic non-linear inductor is the following:

$$
u(t)=\frac{d \lambda(i(t))}{d t}
$$

where $u, i$ are the voltage and current in the bipole, and $\lambda(i(t))$ indicates the magnetic flux linkage as function of the current. The instantaneous link between the flux linkage and the current can be expressed by the relation $\lambda(i(t))=L_{a}(i(t)) i(t)$, in which the apparent inductance $L_{a}$ is function of the current only. By utilising the chain differentiation rule, it can be expanded as:

$$
\begin{aligned}
u(t) & =\frac{d \lambda(i(t))}{d t}=\frac{d\left(L_{a}(i(t)) i(t)\right)}{d t}= \\
& =\left[L_{a}(i(t))+i(t) \frac{d L_{a}(i(t))}{d i(t)}\right] \frac{d i(t)}{d t}= \\
& =\left[L_{a}(i(t))+L_{d}(i(t))\right] \frac{d i(t)}{d t}= \\
& =L(i) \frac{d i(t)}{d t} .
\end{aligned}
$$

Equation (3) introduces the differential inductance $L_{d}$. The final expression is formally similar to the linear case, with the obvious distinction of an explicit dependence on the current of the instantaneous inductance $L$

$$
L(i)=L_{a}(i)+i(t) \frac{d L_{a}(i)}{d i} .
$$

A graphical interpretation of the apparent and instantaneous inductances is reported in Fig. 1. The instantaneous inductance $L$ represents the actual inductance around the operating point $\left(i_{d c}, \lambda_{d c},\right)$ and it expresses the derivative $d \lambda / d i$ of the flux linkage with respect to the current in that point.

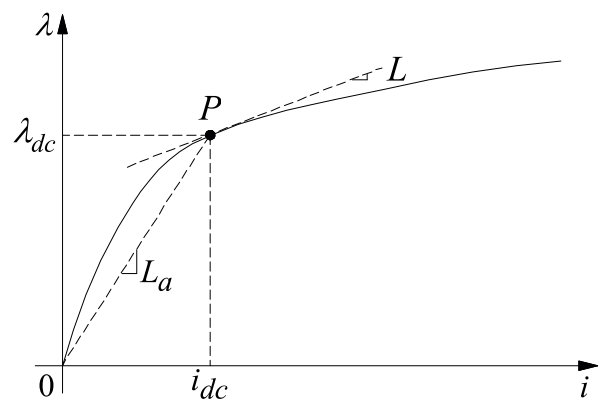

Fig. 1. Graphical interpretation of $L$ and $L_{a}$

\section{B. Inverse- $\Gamma$ equivalent circuit at standstill}

In case of a linear system, the IM mathematical model (1) yields the well-known inverse- $\Gamma$ electrical equivalent circuit [16], which features a transient inductance $L_{t}$ in the stator branch and the magnetising inductance $L_{\varphi}$ in one of the two derived branches (Fig. 2). 


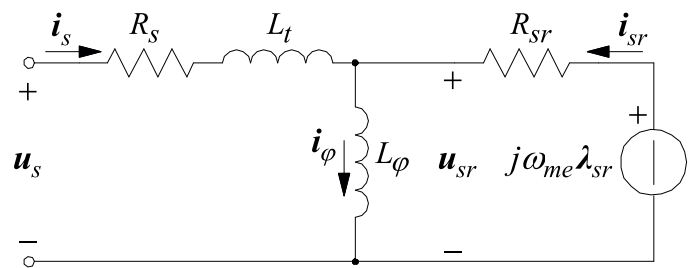

Fig. 2. Inverse- $\Gamma$ equivalent circuit for linear systems.

In case of saturation of the motor magnetic paths, establishing the flux density will require an additional component of the magnetising current $\boldsymbol{i}_{\varphi}$, which will be a nonlinear function of the stator flux. The combined effect of the magnetomotive force (mmf) required by the saturated stator teeth and yoke may be incorporated in the inverse- $\Gamma$ circuit by adding an appropriate nonlinear inductive element across the stator flux linkage ([17]). To maintain the same circuit topology of Fig. 2, the saturation effect has been split into the inductances $L_{t}\left(\boldsymbol{i}_{s}\right)$ and $L_{\varphi}\left(\boldsymbol{i}_{\varphi}\right)$, which then become functions of the respective current space vectors, according to [16].

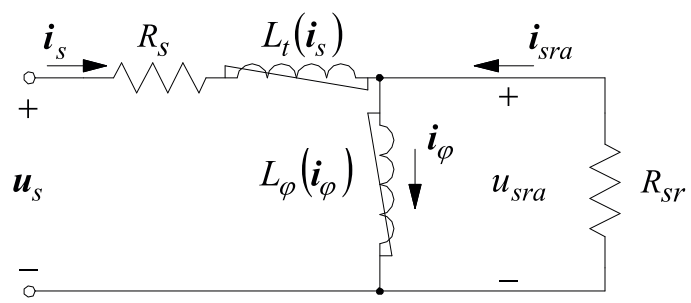

Fig. 3. Inverse- $\Gamma$ vector equivalent circuit including stator iron saturation, at standstill.

At standstill $\left(\omega_{m e}=0\right)$, and consistently with the general discussion of Sect. II-A, which applies also to vector quantities, the extension to the non-linear case can be obtained by expressing the flux linkages derivatives in (1) as functions of the instantaneous inductances:

$$
\left\{\begin{aligned}
\boldsymbol{u}_{s} & =R_{s} \boldsymbol{i}_{s}+L_{t}\left(\boldsymbol{i}_{s}\right) \frac{d \boldsymbol{i}_{s}}{d t}+L_{\varphi}\left(\boldsymbol{i}_{\varphi}\right) \frac{d \boldsymbol{i}_{\varphi}}{d t} \\
\mathbf{0} & =R_{s r} \boldsymbol{i}_{s r}+L_{\varphi}\left(\boldsymbol{i}_{\varphi}\right) \frac{d \boldsymbol{i}_{\varphi}}{d t}
\end{aligned}\right.
$$

where the dependences of the inverse- $\Gamma$ circuit inductances to the current space vectors are made explicit. The resulting equivalent circuit is reported in Fig.3. The voltage balance equation for the phase $a$ is obtained by taking the real components of (5):

$$
\left\{\begin{aligned}
u_{s a} & =R_{s} i_{s a}+L_{t}\left(\boldsymbol{i}_{s}\right) \frac{d i_{s a}}{d t}+L_{\varphi}\left(\boldsymbol{i}_{\varphi}\right) \frac{d i_{\varphi a}}{d t} \\
0 & =R_{s r} i_{s r a}+L_{\varphi}\left(\boldsymbol{i}_{\varphi}\right) \frac{d i_{\varphi a}}{d t}
\end{aligned}\right.
$$

Clearly, further simplifications to (6) are possible only if either the system is linear, so that inductances are independent to the currents, or in the particular case in which one phase is disconnected, which realises the conditions

$$
\begin{gathered}
i_{s b}=-i_{s a}, \quad i_{s c}=0 \\
i_{\varphi b}=-i_{\varphi a}, \quad i_{\varphi c}=0
\end{gathered}
$$

and, of course, $u_{s b}=-u_{s a}, u_{s c}=0$. If this is the case, equations (6) collapse to the following:

$$
\left\{\begin{aligned}
u_{s a} & =R_{s} i_{s a}+L_{t}\left(i_{s a}\right) \frac{d i_{s a}}{d t}+L_{\varphi a}\left(i_{\varphi a}\right) \frac{d i_{\varphi a}}{d t} \\
0 & =R_{s r} i_{s r a}+L_{\varphi}\left(i_{\varphi a}\right) \frac{d i_{\varphi a}}{d t}
\end{aligned}\right.
$$

The per-phase equivalent circuit, including saturation effects, which will be the reference for the next Sections, is reported in Fig. 4. It is worth to recall that due to the setting $\omega_{m e}=0$, the representation is valid at standstill only.

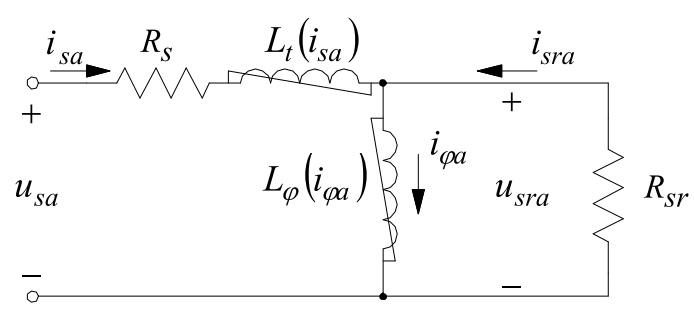

Fig. 4. Per-phase inverse- $\Gamma$ equivalent circuit at standstill, including saturation.

According to Sect. II-A, the inductances of the circuit are all of instantaneous type and each of them depends on the current that flows in its own circuit branch; in case of absence of saturation, their differential component is null, and they reduce to the apparent (and constant) inductance type. Once more, it is worth to highlight that the instantaneous inductances are suitable for writing voltage balance equations, as in (8). The conventional nomenclature still holds, referring to $L_{\varphi}$ and $L_{t}$ as magnetising and transient (or total leakage) inductances respectively. The equation set (6) is the base for the proposed procedure of parameters estimation. As mentioned, the aim is to make a set of measurements with different test signals, and at different current levels, wanting to get the link between the electrical parameters of Fig.3 and the different operating conditions. Those parameters are essential in most of the sensorless algorithms reported in literature, as for example thus related to the model reference adaptive systems (MRAS) class. In the following, the focus will be brought to the derivation of the four-parameter set under the hypothesis (7), but the results can be readily extended to the case of a current vector positioned anywhere in the stationary reference frame, by the use of (6) and the vector version of (4).

\section{THEORY OF OPERATIONS}

The estimation procedure refers to the model of Fig. 4 and it consists of four consecutive steps, each exploiting the results of the preceding one. The parameters are estimated by imposing a known voltage and measuring the current in a single phase (let it be phase $a$ ). The constraint on the voltages which makes valid the representation at standstill 


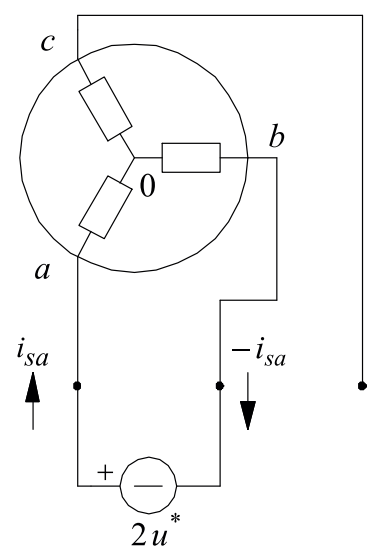

(a)

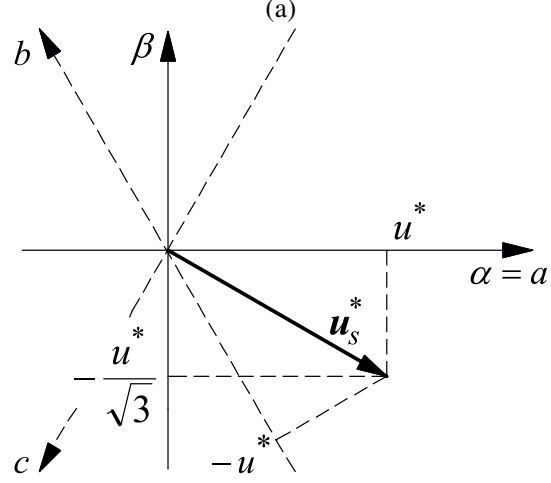

(b)

Fig. 5. The selected topology for induction motor voltage supply. (a) One phase open. (b) Suitable voltage SVM reference.

$\left(u_{s b}=-u_{s a}, u_{s c}=0\right)$ is easily obtained by the topology reported in Fig. 5a.

The voltage generator of Fig. 5a is computed to get a net phase voltage (referred to the motor neutral point 0) $u_{s a}=$ $u_{a 0}=u^{*}$. When the IM is fed by a conventional three-phase space-vector-modulated (SVM) inverter, the candidate voltage vector reference is the following:

$$
\boldsymbol{u}_{s}^{*}=\frac{2}{3}\left(u^{*}-u^{*} e^{j 2 \pi / 3}\right)
$$

It is easy to see that (9) implements the topology of Fig. 5(a). The voltage vector $\boldsymbol{u}_{s}^{*}$ is obtained by applying $u^{*}$ to the phase $a,-u^{*}$ to the phase $b$ and a null voltage to the phase $c$. The current flows from phase $a$ to $b$, while $c$ remains virtually open. In the stationary frame, the reference space vector is then $u^{*}-j u^{*} / \sqrt{3}$, as shown in Fig. 5(b).

As a relevant add-on feature, the motor will remain connected to the plant with unmodified electrical and mechanical connections, and this fits for a specific requirement of many industrial applications.

\section{A. Stator resistance estimation}

The first parameter to be estimated is the stator resistance $R_{s}$. A DC current is imposed in the circuit of Fig. 4 by setting $u^{*}=U_{s a, d c}$ in (9). The inductances in Fig. 4 behave as short-circuits and no current flows into the $R_{s r}$ branch. Consequently, the ratio between the phase voltage and current returns the expected estimation of the $R_{s}$ value.
Two major issues are worth consideration when performing the stator resistance estimation, the precision and the meaning of the results. The former is related to the precision by which $u^{*}$ is known. A voltage sensor is usually avoided and the phase voltage is approximated by its reference (9), provided that an adequate compensation of inverter non-linearities is performed [9]. Further details will be provided in the Sect. IV.

The estimation meaning depends on whether the voltage is measured on motor or inverter terminals. In the first case, the estimated resistance value coincides exactly with the phase resistance $R_{s}$. In the present case, which makes use of the voltage references, the $U_{s a, d c} / I_{s a, d c}$ ratio encompasses the whole chain of resistive elements, including cables and IGBTs on-state resistances. This approach fits for sensorless control algorithms, since they also use the voltage references as input to their motor models.

\section{B. Transient inductance estimation}

The parameter $L_{t}$ is estimated by means of the injection of a high frequency sinusoidal test signal. At steady state, the total impedance of the circuit in Fig. 4 is

$$
Z\left(j \Omega_{h}\right)=R_{s}+j \Omega_{h} L_{t}+\frac{j \Omega_{h} L_{\varphi} R_{s r}}{j \Omega_{h} L_{\varphi}+R_{s r}}
$$

where $\Omega_{h}=2 \pi f_{h}$, with $f_{h}$ frequency of the injected signal. It is recognised that the last term of (10) converges to $R_{s r}$ as the frequency increases. Consequently, for sufficiently high values of $\Omega_{h}$ the following approximation applies:

$$
Z\left(j \Omega_{h}\right) \approx R_{s}+R_{s r}+j \Omega_{h} L_{t}
$$

In principle, the real part of the impedance could be used for the estimation of $R_{s}+R_{s r}$. Since $R_{s}$ is already known, the value $R_{s r}$ could be evaluated. However, this way is not advisable, due to skin and proximity effects that increase the resistances at high frequency, giving fake results for the estimation [18]. On the contrary, the imaginary part gives a straightforward estimate of $L_{t}$.

As the frequency of the test signal increases, the error between the measured $\Im\left[Z\left(j \Omega_{h}\right)\right] / \Omega_{h}$ and $L_{t}$ becomes negligible. For a IM with a rated frequency of $50 \mathrm{~Hz}$, a test angular frequency $\Omega_{h} \geq 2 \pi 300 \mathrm{rad} / \mathrm{s}$ is appropriate.

Unfortunately, the validity of the aforementioned method is restricted to the linear case only. Even if the transient inductance is mainly associated to a leakage flux linkage, nevertheless it may suffer of some saturation effects. It means that the linearity is lost and the impedance (10) makes no sense, since the current is distorted.

Here, a more general approach is proposed. It consists in applying a small sinusoidal voltage $u_{s a, a c}(t)=U_{s a, a c} \cos \left(\Omega_{h} t\right)$ superimposed to a predefined set of increasing DC voltage levels, while measuring the phase currents at each stage. The DC component is selected so to span the whole current range on the basis of the previous knowledge of $R_{s}$, and it sets the working point (as for example the point $P$ of Fig. 1). The low amplitude nature of the AC signal permits the exploitation of the small-signal theory, with supposed linearity around an operating point. This approach is indicated as DC+AC 
method, and it represents a marked difference with respect to the procedure described in [12] where only an AC method at various current levels is exploited.

$\mathrm{AC}$ voltages and currents are used to compute the instantaneous inductance $L_{t}$. The voltage amplitude and frequency are imposed, and thus they are known a priori. The real and imaginary components of the fundamental $\mathrm{AC}$ current phasor $\dot{I}_{s a, a c}=I_{s \alpha}+j I_{s \beta}$ are provided by the Goertzel algorithm, synchronised to the phasor $\dot{U}_{s a, a c}$ of the applied voltage. The Goertzel algorithm is a novel alternative to [12]. It is a singleharmonic discrete Fourier transformation that is particularly suitable for the on-line detection of one or few harmonics (see [19] and Appendix B for details). By construction, it is $\dot{U}_{s a, a c}=U_{s \alpha}+j 0=U_{s a, a c}+j 0$ and from (11) it is:

$$
\begin{aligned}
L_{t} & =\frac{\Im[Z]}{\Omega_{h}}=\frac{1}{\Omega_{h}} \Im\left[\frac{U_{s \alpha}+j 0}{I_{s \alpha}+j I_{s \beta}}\right] \\
& =-\frac{1}{\Omega_{h}} \frac{U_{s \alpha} I_{s \beta}}{I_{s \alpha}^{2}+I_{s \beta}^{2}}
\end{aligned}
$$

A simulation may help in understanding the error that would be introduced by neglecting the saturation effect. Let us consider a simple $R$ - $L$ circuit where $R=1 \Omega$ and $L$ is a (known) non-linear function of the current (Fig. 6). As mentioned, the proposed DC+AC method imposes a DC voltage to set different operating points, and a superimposed AC voltage of $2 \mathrm{~V}$ at $300 \mathrm{~Hz}$ to detect the instantaneous inductance, by means of expression (12). Results are shown in Fig. 6. The proposed estimation is able to track precisely the

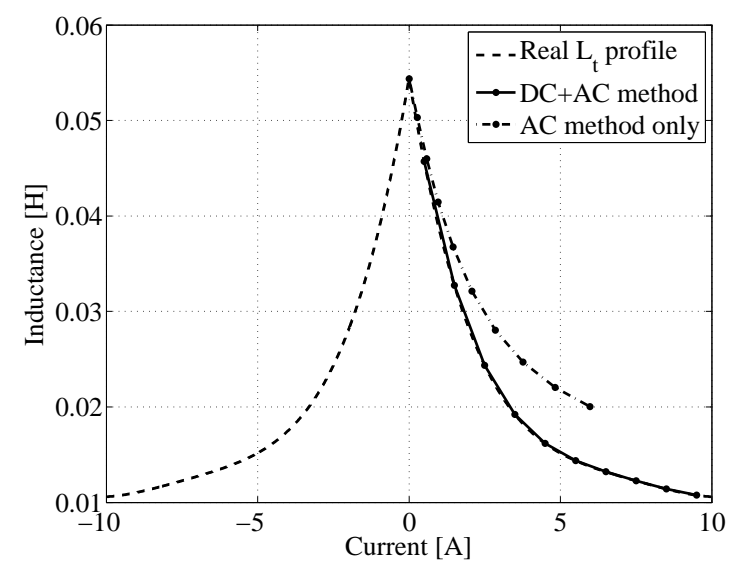

Fig. 6. Effect of $L_{t}$ saturation in the accuracy of the estimation methods.

original (known) outline of the saturated inductance, while the conventional AC method, which applies only AC voltages of increasing amplitudes, clearly fails even for medium current levels.

\section{Magnetising inductance estimation}

The $L_{\varphi}$ estimation at standstill is based on the model discussed in Sect. II-B. The proposed method consists of two steps: $a$ ) impose a DC current level $I_{s a, d c}$, to fix a steady state working point; $b$ ) set a null voltage vector and integrate the resistive component of the current, until the system reaches the null state.
In the first step, when the inverse- $\Gamma$ circuit of Fig. 4 is fed by a DC current, the inductances are short-circuits and the magnetic energy is stored without being transformed into mechanical energy. The flux linkages are obtained by integrating the equation (8) using the definition (3):

$$
\begin{aligned}
& \int_{0}^{t}\left(u_{s a}-R_{s} i_{s a}\right) d t=u_{s a}(0)-R_{s} i_{s a}(0)+ \\
& +\lambda_{t a}(t)-\lambda_{t a}(0)+\lambda_{\varphi a}(t)-\lambda_{\varphi a}(0)
\end{aligned}
$$

At $t=0$ the circuit is in a steady state DC condition, and therefore $u_{s a}(0)-R_{s} i_{s a}(0)=0$. Afterwards, a null voltage $u_{s a}(t)=0$ is applied to the motor (step $\left.b\right)$ ) and both the current and the motor flux linkages converge to zero, so that

$$
\lim _{t \rightarrow+\infty} \int_{0}^{t}\left(u_{s a}-R_{s} i_{s a}\right) d t=-\lambda_{t a}(0)-\lambda_{\varphi a}(0)=-\lambda_{s a}(0)
$$

At $t=0$ the currents in the two inductances of Fig. 4 are the same $\left(i_{s a}(0)=i_{\varphi a}(0)=I_{s a, d c}\right)$ and they are linked to the flux linkages by the apparent inductances, as outlined in Sect. II-A. Merging this information with (14) gives:

$$
\begin{aligned}
& L_{a}\left(I_{s a, d c}\right)=L_{\varphi a}\left(I_{s a, d c}\right)+L_{t a}\left(I_{s a, d c}\right)= \\
& =-\frac{1}{I_{s a, d c}} \lim _{t \rightarrow \infty} \int_{0}^{t}\left(u_{s a}-R_{s} i_{s a}\right) d t
\end{aligned}
$$

The expression (15) suggests the way to compute the magnetising apparent inductance $L_{\varphi a}$, which will be used in conjunction with (4) to calculate the instantaneous inductance $L_{\varphi}$. There is an obvious advantage in considering (15), since it is not affected by inductance saturation, allowing different DC current values $I_{s a, d c}$ to be set in order to map $\lambda_{\varphi a}$, and $L_{\varphi a}$ immediately after, as function of the current.

As mentioned earlier, the computation requires the knowledge of both the resistance $R_{s}$ and the transient inductance $L_{t}$, which at this point are available from the previous steps. An effective procedure consists in prefiguring the total flux linkage $\lambda_{s a}(i)=\lambda_{\varphi a}(i)+\lambda_{t a}(i)$ as a third-order polynomial function of the current $I_{s a, d c}([10]$, [20]) so that

$$
\begin{aligned}
& L_{a}(i)=\frac{\lambda_{s a}(i)}{i}=\frac{p_{3} i^{3}+p_{2} i^{2}+p_{1} i+p_{0}}{i}= \\
& =p_{3} i^{2}+p_{2} i+p_{1}+p_{0} i^{-1}
\end{aligned}
$$

The coefficients $\left(p_{3}, p_{2}, p_{1}, p_{0}\right)$ can be obtained by a polynomial fitting algorithm, which elaborates the measurements of $\lambda_{s a}$ obtained from (14) by imposing different $I_{s a, d c}$ current levels. Substituting the last of (16) in (4) returns

$L(i)=L_{a}(i)+i \frac{d L_{a}(i)}{d i}=3 p_{3} i^{2}+2 p_{2} i+p_{1}=L_{\varphi}(i)+L_{t}(i)$

that will be used to get $L_{\varphi}$ from the value of $L_{t}$ obtained in Sect. III-B.

In principle, the main drawback of expression (15) relies in the digital implementation of a pure integrator. This is a rather annoying and well-known problem, due to the DC offset that always affects the integrator input, causing output drift. Effective countermeasures, taylored on the singularity of the proposed method, are discussed in Sect. IV. 


\section{Rotor resistance estimation}

The rotor resistance referred to the stator $R_{s r}$ (Fig. 4) can be obtained by exciting the system with a sinusoidal, lowfrequency and low-amplitude voltage $u_{s a}=U_{s a} \cos \left(\Omega_{l} t\right)$. Similarly to Sect. III-B and for an effective mathematical elaboration, a $\alpha-\beta$ reference frame fixed to the phasor $\dot{U}_{s a}$ is chosen, so that the sinusoidal phasor $\dot{I}_{s a}=I_{s \alpha}+j I_{s \beta}$ can be extracted fairly easily by the Goertzel algorithm, which represents a novel and distinctive feature of the proposed method over the existing ones, as [14].

The voltage phasor $\dot{U}_{s r a}=U_{s r \alpha}+j U_{s r \beta}$ across $R_{s r}$ is given by:

$$
\begin{aligned}
\dot{U}_{s r a} & =\dot{U}_{s a}-\left(R_{s}+j \Omega_{l} L_{t}\right) \dot{I}_{s a}= \\
& =U_{s \alpha}-\left(R_{s}+j \Omega_{l} L_{t}\right)\left(I_{s \alpha}+j I_{s \beta}\right)
\end{aligned}
$$

The resistance value is the ratio between the voltage and current amplitudes:

$$
R_{s r}=\frac{\left|\dot{U}_{s r a}\right|}{\left|\dot{I}_{s r a}\right|}
$$

Due to the resistive nature of the branch, the current phasor $\dot{I}_{s r a}$ is the component of $\dot{I}_{s a}$ in phase with $\dot{U}_{s r a}$ :

$$
\dot{I}_{s r a}=\dot{I}_{s a} \cos \left(\vartheta_{s r}\right)
$$

where $\vartheta_{s r}$ is the angle between $\dot{I}_{s a}$ and $\dot{U}_{s r a}$. The value of $\cos \left(\vartheta_{s r}\right)$ can be obtained by the reverse expression of the scalar product between $\dot{I}_{s a}$ and $\dot{U}_{s r a}$, as follows:

$$
\cos \left(\vartheta_{s r}\right)=\frac{U_{s r \alpha} I_{s \alpha}+U_{s r \beta} I_{s \beta}}{\left|\dot{U}_{s r a}\right|\left|\dot{I}_{s a}\right|}
$$

Substituting expression (21) in (19) gives:

$$
R_{s r}=\frac{U_{s r \alpha}^{2}+U_{s r \beta}^{2}}{U_{s r \alpha} I_{s \alpha}+U_{s r \beta} I_{s \beta}}
$$

which is the resolutive equation for the estimation of $R_{s r}$.

Different frequencies are used to map the $R_{s r}$ value in the range of interest, which is usually from zero to the IM nominal slip frequency $(5 \% \div 10 \%$ of the nominal IM speed). Frequencies higher than the nominal slip speed are not meaningful because they do not fall under normal the workin conditions of a vector-controlled IM drive.

\section{EXPERIMENTAL RESULTS}

A series of experimental results were performed on an IM whose rated values and nameplate data are reported in Appendix A. Experiments were carried out with a space-vector modulation of $10 \mathrm{kHz}$, IGBT dead times of $4 \mu$ s and a DC bus of $100 \mathrm{~V}$, although results are not critically affected by an increased value of the DC-bus voltage.

\section{A. Stator resistance estimation}

As illustrated in Sect. III-A, a DC test is performed to estimate the stator resistance. It is known that the deadtime negatively affects the phase voltage generation of the PWM, introducing an ideally-constant voltage drop whose sign depends on the sign of the phase current [21]. It is also well known [13] that the voltage distortion behaviour at low current levels diverges from the theoretical profile, being a constant only for sufficiently high current magnitudes.

The non linear behaviour of the inverter can be mitigated by prefiguring an input-output mapping. To this aim, different DC current levels $I_{s a, d c}$ are first applied to the motor, and the corresponding voltage references $u_{s a}^{*}$ are recorded accordingly. Fig. 7 shows the static $u_{s a}^{*}=f\left(I_{s a, d c}\right)$ curve measured in laboratory.

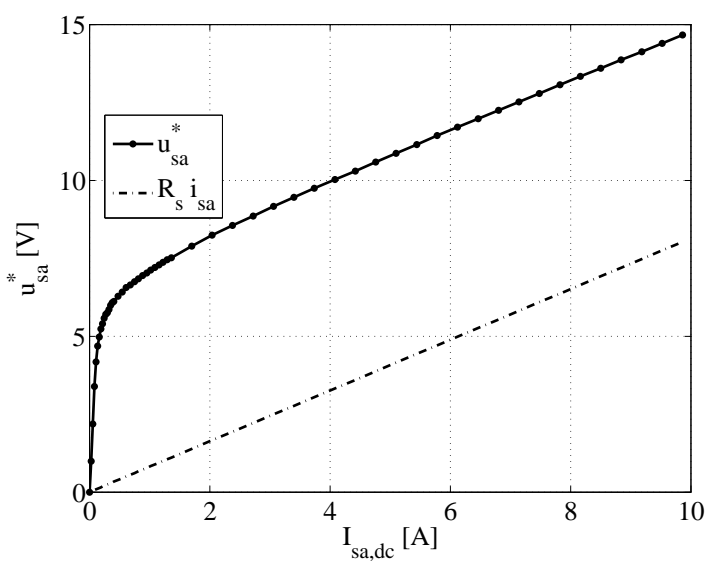

Fig. 7. Static $u_{s a}=f\left(i_{s a}\right)$ for $R_{s}$ estimation.

The curve accounts for both the voltage distortion effects at low current levels and the constant voltage drop at higher current levels. The curve of Fig. 7 can be profitably used for an estimation of $R_{s}$, provided that the derivative is computed on the higher-current part only. In this work, the last five $u_{s a}^{*}$ values were used with the corresponding $I_{s a, d c}$ samples, and then processed through a simple least-square method to obtain the estimation of $R_{s}$. The final result was $R_{s}=0,814 \Omega$, confirmed by an off-line DC test with a high precision multimeter (Agilent 34401A). Of course, the resistance varies with the temperature and, in case of sensorless control applications, on-line tracking would be highly recommended.

Fig. 7 also reports the ideal $R_{s} I_{s a, d c}$ curve, that is, the portion which is directly related to the motor and cables linear resistive elements. The difference between the ideal and the measured curve represents the non-linear elements, and it is used to compensate the voltage reference values generated by the control algorithm (via a look-up table or a polynomial approximation), to obtain a close match between the reference and the real phase voltage. In absence of voltage sensors, the outlined procedure has revealed good usability and it was essential to perform the subsequent steps of the estimation procedure.

\section{B. Transient inductance estimation}

The second step of the procedure, as discussed in Section III-B, estimates the transient inductance $L_{t}$. A superimposed AC voltage of $2 \mathrm{~V}$ at $300 \mathrm{~Hz}$ was used to investigate the asymptotic effect of (11). Voltage and current fundamental harmonics were acquired and processed to obtain the imaginary part of the impedance $Z(j \Omega)$. Results are shown in Fig. 8. 


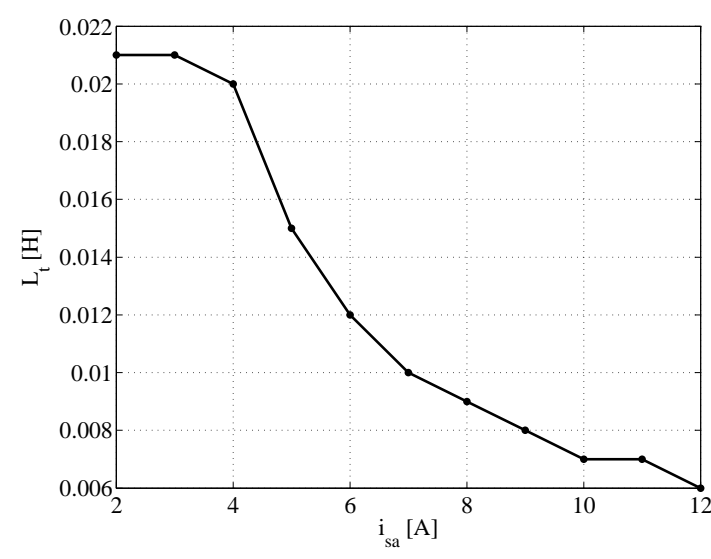

Fig. 8. $L_{t}$ estimation by $\mathrm{DC}+\mathrm{AC}$ method.

The $L_{t}$ profile shows a certain degree of saturation, which could be explained considering the saturation of the stator slot corners in the motor for higher currents, affecting the value of the total leakage flux linkage in the air gap. These measurements confirm that $L_{t}$ is a delicate parameter in an IM model, since its value is very small and difficult to calculate. On the other hand, its impact on sensorless motor models is very low, as documented in [22]. Authors also found that $L_{t}$ values obtained with the proposed procedure are very repeatable by changing the amplitude and/or the frequency of the superimposed AC voltage.

\section{Magnetising inductance estimation}

The experimental measurement of the magnetising inductance was found to be the most challenging part of the procedure. First, a reference curve was obtained by offline measurements, in order to compare the results of the procedure. The data were obtained by measuring the current of the phase $a$ and the differential voltage between the phases $a$ and $b$, at the inverter output. Different transients were imposed by forcing different DC currents in the motor with a DC-power supply. The step-down transient of both current and voltage were recorded and post-processed numerically, calculating the stator resistance before the transient and the integral (13) until the current reached the zero state.

Then, the automatic detection of the magnetising inductance was implemented by feeding the motor with the inverter directly. As mentioned in Sect. III-C, a drawback of the proposed approach relies in the digital implementation of a pure integrator, which suffers of drift problems. Such issues have been addressed in some papers like [23] and [24], but unfortunately proposed solutions do not work in a standstill condition, where a single motor phase is equivalent to a simple $R L$ circuit.

In order to reduce the drift effect, the procedure adopts the following approach. A first transient from zero to a DC current level is performed, by imposing a suitable phaseto-phase DC voltage $\left(u^{*}=U_{s a, d c}\right.$ in Fig. 5). When the DC condition is reached, the average back electromotive force $E_{s a, d c}=U_{s a, d c}-R_{s} I_{s a, d c}$ is calculated. In principle, $E_{s a, d c}$ should be equal to zero but, in practice, the non-zero experimental value represents the unavoidable offset which must be subtracted from the integrator input when calculating the stator flux linkage using (13). The current is then forced again to zero by imposing a zero voltage, and calculating the integral (13) over the whole transient. Different transients are repeated for different DC current levels, in order to get the profile of the integral in the whole IM current range. The curve was then compared with the reference one obtained with the DC-power supply.

Two important aspects must be considered while performing the automatic detection of the magnetising inductance as function of the stator current. Such aspects have not been previously considered in [13] and are meant to improve the overall accuracy of the procedure.

The first is related to the presence of the voltage drop $u_{t}$ caused by the IGBTs and the threshold voltage $u_{d}$ of the free-wheeling diodes within the inverter. During a step-down transient of the current $i_{s a}$ from positive values to zero, the topology outlined in Fig. 5a becomes that depicted in Fig. 9.

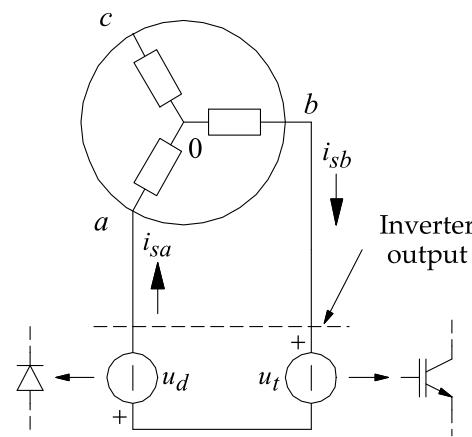

Fig. 9. Step-down current transient scheme.

The phase-to-phase voltage $u_{s a b}$ is obtained by writing twice (one for each phase $a, b$ ) the equation (8), then by taking the difference on both sides with the assumption (7). It is:

$$
u_{s a b}=2 R_{s} i_{s a}+2 \frac{\lambda_{t}\left(i_{s a}\right)}{d t}+2 \frac{\lambda_{\varphi}\left(i_{\varphi a}\right)}{d t}
$$

Kirchoff's second law, applied to the mesh of Fig. 9, returns:

$$
u_{d}+u_{s a b}+u_{t}=0
$$

Substituting (23) in (24), integrating side by side and applying exactly the same considerations carried out in Sect. III-C, one obtains the phase flux linkage $\lambda_{s a}(0)$, computed at time $t=0$ and current $i_{s a}=I_{s a, d c}$ :

$$
\lim _{t \rightarrow+\infty} \int_{0}^{t}\left(\frac{u_{d}+u_{t}}{2}+R_{s} i_{s a}\right) d t=\lambda_{s a}(0)
$$

and then

$$
\begin{aligned}
& L_{a}\left(I_{s a, d c}\right)=L_{\varphi a}\left(I_{s a, d c}\right)+L_{t a}\left(I_{s a, d c}\right)= \\
& =\frac{1}{I_{s a, d c}} \lim _{t \rightarrow \infty} \int_{0}^{t}\left(\frac{u_{d}+v_{t}}{2}+R_{s} i_{s a}\right) d t
\end{aligned}
$$

The value $u_{d}+u_{t}$ can be either inferred from the data sheet or found by means of a dedicated off-line measurement, as specified hereafter. By imposing a voltage step-down transient, 
which forces the current to move from the steady-state rated value to zero, and by measuring the $u_{s a b}$ voltage at the inverter output by a differential probe, a map of $u_{d}+u_{t}$ as function of the current can be obtained. The result, for the present case, is shown in Fig. 10. The profile was approximated by piece-wise continuous lines and implemented into equation (26), for each current sample.

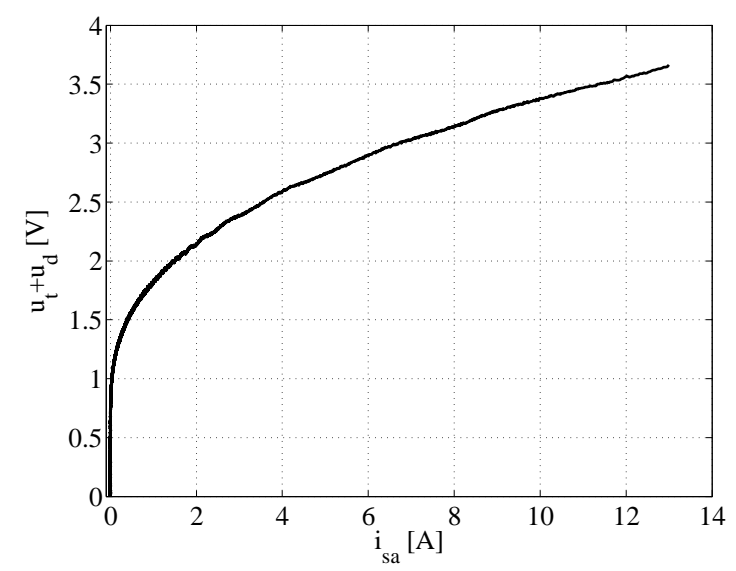

Fig. 10. Profile of the $u_{t}+u_{d}$ curve as function of $i_{s a}$.

The second aspect is related to the accuracy of the current sensor within the inverter. Even if the measurement offset can be removed by simple automatic procedures, low-accuracy sensors could lead to potential wrong results in the flux linkage estimation. To this purpose, the measurements of the inverter built-in Hall current sensor were compared, before the activation of the estimation procedure, to the current measurements obtained with a high-accuracy ammeter (QinetiQ PPA2530). Fig. 11 shows the percentage error of the Hall sensor, normalised to the rated motor current $I_{N}$ (see Table I).

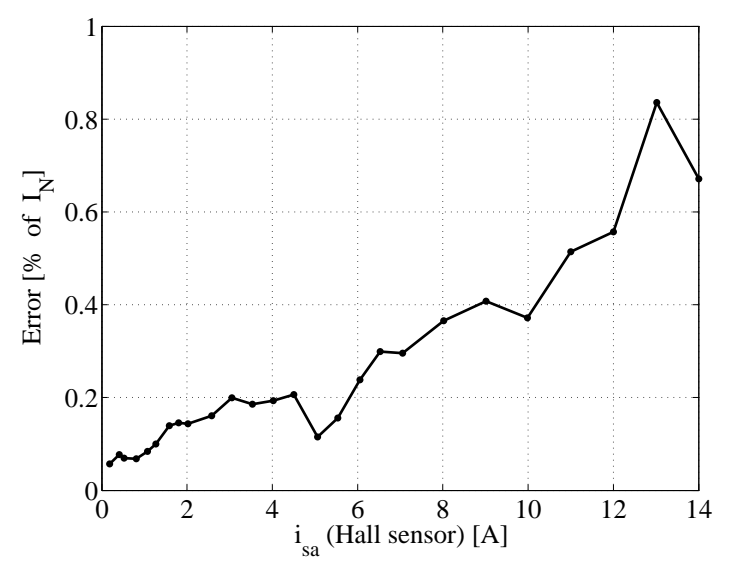

Fig. 11. Percentage error of the current sensor measurement.

The discrepancy increases at higher current levels, potentially affecting the correct estimation of the magnetising inductance. As a rough-and-ready countermeasure, a constant bias of $+0,4 \%$ was added to the Hall sensor measurement for the calculations of the automatic procedures. The simple use of only a constant term was enough to obtain very reliable results.

According to (25), the experimental measurements returned the phase flux linkage $\lambda_{s a}$ as function of the initial steady-state current value, $\lambda_{s a}=\lambda_{s a}\left(I_{s a, d c}\right)$. The profile of the stator flux closely matches the reference one obtained by means of the DC power supply. Only low-current results deviate from the reference profile, because it is difficult to integrate the flux linkage at low current levels. In any case, they can be easily replaced by a linear approximation of the curve, since the flux is within the linear region of the magnetic $B-H$ curve. To have an objective verification of the result, a finite element analysis (FEA) based on the IM geometry and windings data has been carried out, too, as suggested in [25] and [26]. The FEA simulation was performed by imposing growing levels of current density to both phases $a$ and $b$, while setting $i_{c}=0$, to reproduce the conditions of the proposed procedure. The measured and simulated flux linkages are reported in Fig. 12a, while Fig. 12b shows a flux map during computer FEA simulations.

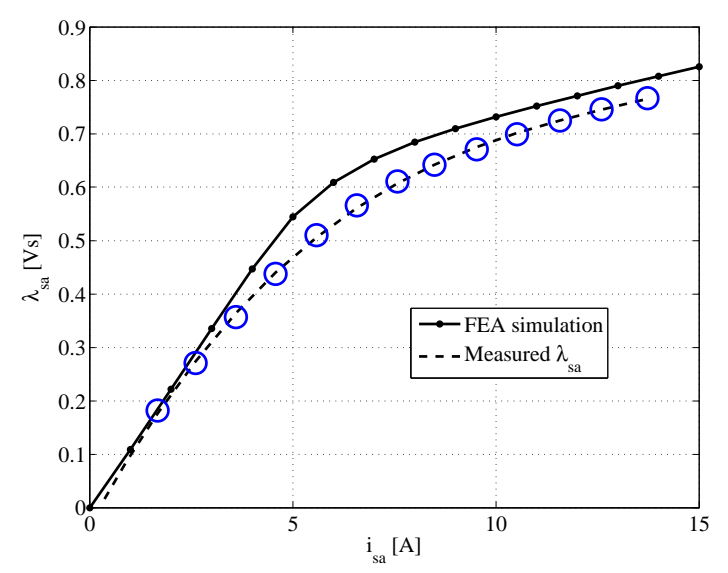

(a)

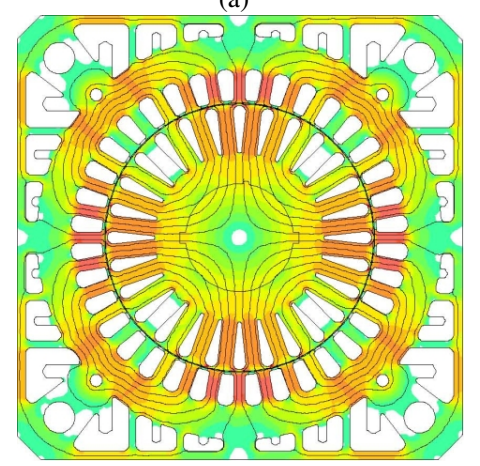

(b)

Fig. 12. (a) $\lambda_{s a}$, measurement and simulation by finite element analysis, (b) IM flux map resulting from FEA simulations.

The error between the measured and FEA-simulated flux linkage remains within $7 \%$ of the rated one, and the small mismatch can be largely attributed to the use of a 2- $D$ (instead of $3-D$ ) finite element analysis tool. The result is therefore quite accurate if one consider that, for example, the analysis carried out in [27] about the influence of parameter variations in sensorless rotor flux oriented IM, shows that a incorrect 
setting of magnetising inductance by $20 \%$ produces a speed detuning of only $0.2 \%$ and a torque error of $8 \%$.

For the sake of completeness, the estimation procedure has been applied to a second motor (fed by the same inverter), whose nameplate data are reported in Table II. The accuracy of the results matches the expectations. In particular, it is to stress out the importance of the correction suggested in Fig. 10 , and the related discussion. The voltage drop across the switching devices cannot be neglected, because it turns out to play a crucial role in the integration (25), as shown in Fig. 13, where the flux linkages (with DC power supply and PWM inverter, respectively, as voltage sources) are compared. The inaccuracy suggests that some countermeasures have to be taken anyway. Of course, the finest the estimation of voltage drops in any working condition, the better the accuracy of flux linkage estimation.

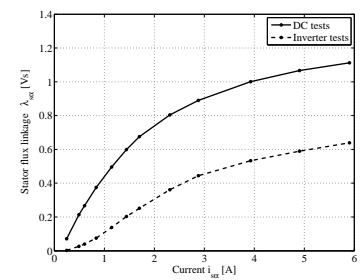

Fig. 13. $\lambda_{s \alpha}$ estimation with DC pwer supply and PWM inverter as voltage sources.

The availability of the flux linkage enables the computation of the magnetising inductance, which can be obtained by using the equations (16) and (17) and the values of $L_{t}$ (Fig. 8). The behaviour of both the sum of the two inductances and the magnetising inductance alone are reported in Fig. 14.

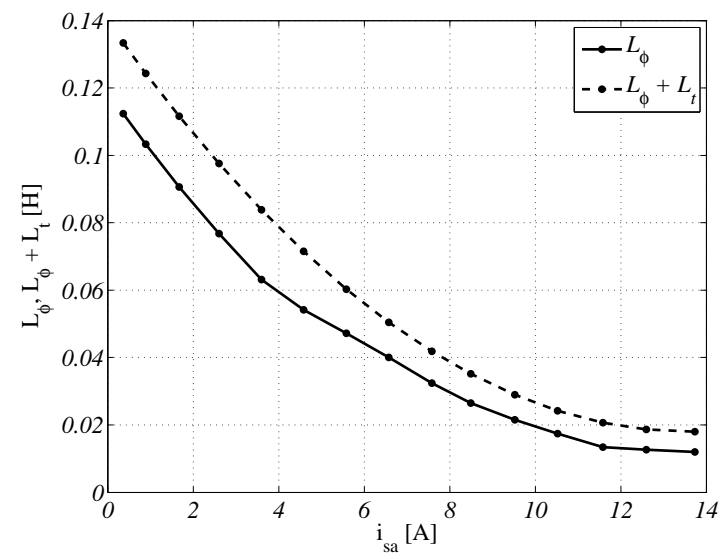

Fig. 14. Computation of $L_{\varphi}$ from $\lambda_{s a}$ and $L_{t}$.

\section{Rotor resistance referred to the stator estimation}

The last part of the procedure estimates the $R_{s r}$ parameter. Since the maximum frequency of interest for the injected sinusoidal signals spans over few Hertz, it would be very easy to force hazardous values of current even with very low voltages. This poses a potential problem, since the estimation of lowamplitude sinusoidal voltages is rather difficult, although the
LUT for inverter non-linearities compensation could give some benefits.

Unlike [14], here the problem is overcome by using the same DC-bias technique presented in Sect. III-B for the estimation of $L_{t}$. In this case, a single bias is used to shift the reference voltage (and the current as well) so that the superimposed sinusoidal voltage are sufficiently far from the near-zero region, where the voltage compensation shows its weakness. Fig. 15 reports the $R_{s r}$ outline as function of the frequency. Meaningful frequencies are from zero to the nominal slip speed $\Omega_{s l N}$, which from the data of Table I corresponds to $2,5 \mathrm{~Hz}$.

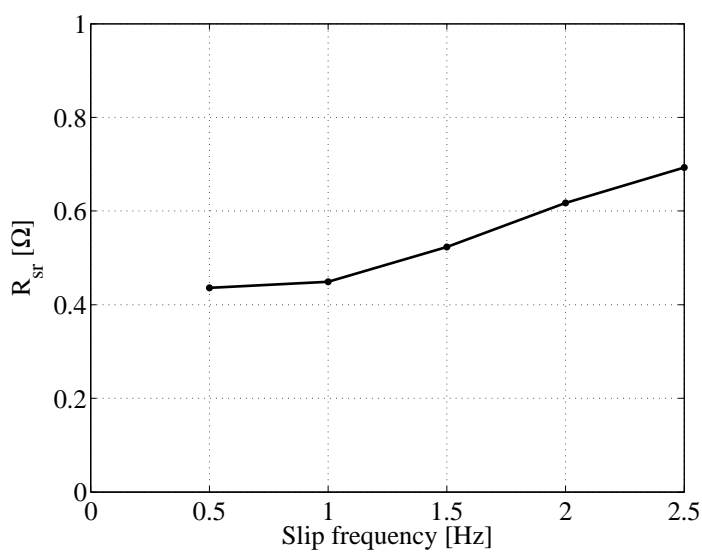

Fig. 15. $R_{s r}$ estimation.

It is evident that the $R_{s r}$ parameter suffers of the skin effect even in the range of interest. It is worth to note that skin effect represents only a part of the $R_{s r}$ increment during normal operation, the other effect being the resistance increase as function of the temperature. Therefore, the proposed procedure is useful for an initial knowledge of the $R_{s r}$ profile, but it is advisable to exploit an on-line $R_{s r}$ tracking procedure to preserve the validity of the model during normal drive operation.

\section{CONCLUSIONS}

In this paper, an automatic procedure for IM parameters estimation was presented. The procedure is performed at complete standstill, which is particularly appreciated when the motor is already connected to the load. The estimation fully identifies the parameters of the inverse- $\Gamma$ model, and it includes the non-linearities of both the motor and the voltage inverter. The estimated parameters are ready-to-use for conventional vector-controlled drives, thus representing a step towards the complete self-commissioning. The procedure has been implemented in laboratory and tested on IM prototypes. The results were validated by comparison with an accurate finite element analysis. Further research activity will include extensive tests on motors of different sizes and power ratings. The focus will be also on the on-line tracking of the temperature and frequency-dependant parameters, as stator and rotor resistances, and of iron losses influence as well. 


\section{ACKNOWLEDGEMENTS}

Authors would like to thank SAEL s.r.l., Torri di Quartesolo (VI), Italy, for its financial support. Special thanks to prof. S. Bolognani, N. Bianchi and dr. A. Rais for their wise and unconditioned support to our research activity and to Dr. L. Alberti, for his great help in FEA analysis.

\section{REFERENCES}

[1] K. B. Nordin, D. W. Novotny, and D. S. Zinger, "The influence of motor parameter deviations in feedforward field orientation drive systems," IEEE Trans. Ind. Appl., vol. 21(4), pp. 1009-1015, 1985.

[2] H. Schierling, "Self-commissioning - a novel feature of modern inverterfed induction motor drives," Proceedings of the IEEE Conference on Power Electronics and Variable Speed Drives, pp. 287-290, 1988.

[3] A. M. Khambadkone and J. Holtz, "Vector-controlled induction motor drive with a self-commissioning scheme," IEEE Trans. Ind. Electron., vol. 38(5), pp. 322-327, 1991.

[4] J.-K. Seok, S.-I. Moon, and S.-K. Sul, "Induction machine parameter identification using pwm inverter at standstill," IEEE Trans. Ind. Electron., vol. 12(2), pp. 127-132, 1997.

[5] A. Wolfram, "Induction motor parameter estimation at standstill by means of advanced signal processing methods," Proceedings of the International Exhibition and Conference for Power Electronics Intelligent Motion Power Quality (PCIM), pp. 105-110, 2006.

[6] Y.-S. Kwon, J.-H. Lee, S.-H. Moon, B.-K. Kwon, C.-H. Choi, and J.-K Seok, "Standstill parameter identification of vector-controlled induction motors using the frequency characteristics of rotor bars," IEEE Trans. Ind. Appl., vol. 45(5), pp. 1610-1618, 2009.

[7] K. Akatsu and A. Kawamura, "Sensorless very low-speed and zerospeed estimations with online rotor resistance estimation of induction motor without signal injection," IEEE Trans. Ind. Appl., vol. 36(3), pp. 764-771, 2000.

[8] J. Maes and J. A. Melkebeek, "Speed-sensorless direct torque control of induction motors using an adaptive flux observer," IEEE Trans. Ind. Appl., vol. 36(3), pp. 778-785, 2000.

[9] J. Holtz and J. Quan, "Sensorless vector control of induction motors at very low speed using a nonlinear inverter model and parameter identification," IEEE Trans. Ind. Appl., vol. 38(4), pp. 1087-1095, 2002.

[10] M. Bertoluzzo, G. S. Buja, and R. Menis, "Self-commissioning of rfo im drives: one-test identification of the magnetization characteristic of the motor," IEEE Trans. Ind. Appl., vol. 37(6), pp. 1801-1806, 2001.

[11] A. B. Proca and A. Keyhani, "Identification of variable frequency induction motor models from operating data," IEEE Trans. Energy Convers., vol. 17(1), pp. 24-31, 2002

[12] R. J. Kerman, J. D. Thunes, T. M. Rowan, and D. W. Schlegel, "A frequency-based determination of transient inductance and rotor resistance for field commissioning purposes," IEEE Trans. Ind. Appl., vol. 32(3), pp. 577-584, 1996

[13] M. Ruff, A. Bünte, and H. Grotstollen, "A new self-commissioning scheme for an asynchronous motor drive system," Conference Record of the IEEE Industry Applications Society (IAS) Annual Meeting, vol. 1, pp. 616-623, 1994.

14] J. Godbersen, "A stand-still method for estimating the rotor resistance of induction motors," Conference Record of the IEEE Industry Applications Society (IAS) Annual Meeting, pp. 900-905, 1999.

[15] A.Lamine and E.Levi, "Dynamic induction machine modelling considering the stray load losses," Universities Power Engineering Conference, vol. 2, pp. 582-586, 2004.

[16] P. Vas, Vector Control of AC Machines. New York, USA: Oxford University Press, 1990.

[17] G. R. Slemon, Electric Machines and Drives. New York, USA: Addison Wesley, Inc., 1992.

[18] T. J. White and J. C. Hinton, "Compensation for the skin effect in vector-controlled induction motor drive systems," Seventh International Conference on Electrical Machines and Drives, pp. 301-305, 1995.

[19] A. V. Oppenheim and R. W. Schafer, Discrete-Time Signal Processing, Third Edition. Upper Saddle River, NJ, USA: Prentice-Hall, Inc., 2009.

[20] M. Ruff and H. Grotstollen, "Identification of the saturated mutual inductance of an asynchronous motor at standstill by recursive least squares algorithm," Conference Record of the 5th European Conference on Power Electronics and Applications, vol. 5, pp. 103-108, 1993.

[21] A. R. Munoz and T. A. Lipo, "On-line dead-time compensation technique for open-loop PWM-VSI drives," IEEE Trans. Power Electron., vol. 14(4), pp. 683-689, 1999.
[22] S. Bolognani, L. Peretti, and M. Zigliotto, "Parameter sensitivity analysis of an improved open-loop speed estimate for induction motor drives,' IEEE Trans. Power Electron., vol. 23(4), pp. 2127-2135, 2008.

[23] J. $\mathrm{Hu}$ and $\mathrm{B}$. Wu, "New integration algorithms for estimating motor flux over a wide speed range," IEEE Trans. Power Electron., vol. 13(5), pp. 969-977, 1998.

[24] R. Bojoi, P. Guglielmi, and G. Pellegrino, "Sensorless direct field oriented control of three-phase induction motor drives for low cost applications," Conference Record of the 41st Industry Application Society (IAS) Annual Meeting, vol. 2, pp. 866-872, 2006.

[25] L.Alberti, N.Bianchi, and S. Bolognani, "A very rapid prediction of im performance combining analytical and finite-element analysis," IEEE Trans. Ind. Appl., vol. 44(5), pp. 1505-1512, 2008.

[26] L.Alberti, N.Bianch, and S. Bolognani, "Variable-speed induction machine performance computed using finite-element," IEEE Trans. Ind. Appl., vol. 47(2), pp. 789-797, 2011.

[27] E.Levi and M.Wang, "Impact of parameter variations on speed estimation in sensorless rotor flux oriented induction machines," IEE Conference on Power Electronics and Variable Speed Drives, vol. 456 pp. 305-310, 1998.

\section{APPENDIX A \\ IM NAMEPLATE DATA}

Table I

IM MOTOR PARAMETERS

\begin{tabular}{|c|c|}
\hline Nominal power $P$ & $3,7 \mathrm{~kW}$ \\
\hline Nominal speed $\Omega_{m N}$ & $1500 \mathrm{rpm}$ \\
\hline Nominal current $I_{N}$ & $11,8 A_{\text {eff }}$ \\
\hline Nominal voltage $V_{N}$ & $280 V_{e f f}$ \\
\hline Nominal slip speed $\Omega_{s l N}$ & $150 \mathrm{rpm}$ \\
\hline $\cos (\varphi)$ & 0,77 \\
\hline
\end{tabular}

Table II

SECOND IM MOTOR PARAMETERS

\begin{tabular}{|c|c|}
\hline Nominal power $P$ & $2,2 \mathrm{~kW}$ \\
\hline Nominal speed $\Omega_{m N}$ & $1500 \mathrm{rpm}$ \\
\hline Nominal current $I_{N}$ & $5,1 A_{\text {eff }}$ \\
\hline Nominal slip speed $\Omega_{s l N}$ & $110 \mathrm{rpm}$ \\
\hline
\end{tabular}

\section{APPENDIX B}

\section{GOERTZEL ALGORITHM DETAILS}

The derivation of the Goertzel algorithm for single-tone detection starts from the normalised definition of the discrete Fourier transform (DFT):

$$
X(k)=\sum_{r=0}^{N-1} x(r) W_{N}^{k r} \quad 0 \leq k \leq N-1
$$

where $N$ is the number of samples, $W_{N}=e^{-j 2 \pi / N}, x(r)$ and $X(k)$ are the sampled signal (normalised sample time $T=1$ ) and its frequency-domain transformation (sample frequency 
$F=1 / N)$, respectively. The Goertzel algorithm is obtained by manipulating (27), considering the following identity:

$$
W_{N}^{-k N}=e^{(j 2 \pi / N) N k}=e^{j 2 \pi k}=1
$$

Using (28) into the first of (27), it follows:

$$
X(k)=W_{N}^{-k N} \sum_{r=0}^{N-1} x(r) W_{N}^{k r}=\sum_{r=0}^{N-1} x(r) W_{N}^{-k(N-r)}
$$

Based on (29), the following sequence can be introduced:

$$
y(n)=\sum_{r=0}^{N-1} x(r) W_{N}^{-k(n-r)}
$$

The sequence (30) is equal to $X(k)$ when $n=N$. It represents a convolution between $x(n)$ and $W_{N}^{-k n}$ or, equivalently, the output of a linear system whose impulse response is equal to $W_{N}^{-k n}$. The Z-transform of $W_{N}^{-k n}$ is:

$$
H(z)=\underbrace{\frac{1}{1-2 \cos (2 \pi k / N) z^{-1}+z^{-2}}}_{\text {IIR filter }} \underbrace{\left(1-W_{N}^{k} z^{-1}\right)}_{\text {FIR filter }}
$$

Expression (31) represents the transfer function between the sequence $y(n)$ and the input $x(n)$, split into an IIR filter and a FIR filter. As regards the former, let $s(n)$ be the output of the IIR filter and $S(z)$ its Z-domain value. It follows:

$$
X(z)=\left(1-2 \cos (2 \pi k / N) z^{-1}+z^{-2}\right) S(z)
$$

Back into the time domain, it is:

$$
s(n)=x(n)+2 \cos (2 \pi k / N) s(n-1)-s(n-2)
$$

With the input $x(n)$ being sampled during real-time control, the sequence $s(n)$ is computed online. As regards the FIR filter, its output $y(n)$ is computed only for $n=N$, provided that $s(N)$ and $s(N-1)$ have been previously saved:

$$
X(k)=\left.y(n)\right|_{n=N}=s(N)-W_{N}^{k} s(N-1)
$$

Since $W_{N}^{k}$ is a complex number, the result of (34) is complex, too. The real and imaginary parts are:

$$
\begin{aligned}
& \Re[X(k)]=s(N)-\cos (2 \pi k / N) s(N-1) \\
& \Im[X(k)]=\sin (2 \pi k / N) s(N-1)
\end{aligned}
$$

Since $x(n)$ is a real signal, (33) shows that the computation of $s(n)$ requires two additions and one multiplication, since $2 \cos (2 \pi k / N)$ is stored as a coefficient. Thus, $s(N)$ is obtained with $N$ multiplications and $2 N$ additions. The calculation of $\Re[X(k)]$ and $\Im[X(k)]$ requires two multiplications and one addition. Altogether, the Goertzel algorithm then requires $N+2$ multiplications and $2 N+1$ additions. This is a net saving, if compared to the DFT in (27) which needs $2 N$ multiplications and $2 N$ additions to compute a real and imaginary parts of $X(k)$ from a real signal $x(k)$ [19]. However, the real advantage of the Goertzel algorithm for single harmonic analysis resides in its recursive definition (33). The $N+2$ multiplications and $2 N+1$ additions are spread over the whole time window, and $s(n)$ is updated with one multiplication and two additions in each sample period. In this way, the algorithm can be effectively used in real time without affecting the execution time of control routines. Moreover, the value of $N$ can be increased as needed with no effect on the computational time requirements, obtaining a very high DFT selectivity.

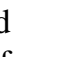

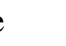

